\title{
Estudio del proceso de desnitrificación en un reactor SBR alimentado con el efluente de un reactor anaerobio de membranas sumergidas SAnMBR.
}

\author{
Study of denitrification process in SBR reactor fed \\ with the effluent of an anaerobic submerged membrane \\ bioreactor SAnMBR.
}

Javier E. Sánchez Ramírez ${ }^{1}$, Alberto Bouzas ${ }^{2}$, Aurora Seco $^{3}$ y $\mathbf{M}^{\mathrm{a}}$ Francisca García Usach ${ }^{4}$.

\begin{abstract}
Resumen
El tratamiento o postratamiento de efluentes anaerobios sugiere del estudio de diversos procesos que permitan la eliminación o remoción de los contaminantes presentes. El objetivo de este trabajo consistió en el estudio de la capacidad de desnitrificación en condiciones anóxicas utilizando los distintos dadores de electrones, disponibles en el efluente del reactor SAnMBR tales como: ácidos grasos volátiles, metano disuelto y sulfuro. La importancia de este estudio radica en la posibilidad de utilizar los ácidos grasos volátiles, el metano y el sulfuro disuelto como dadores de electrones, para la eliminación de nitrógeno. El metano disuelto es una fuente de carbono muy barato y un gas de efecto invernadero eficaz, siendo necesaria su eliminación. El efluente del reactor SAnMBR contiene concentraciones importantes de amonio, fósforo, sulfuro, ácido acético, elementos traza y metano disuelto. Durante la operación del reactor SBR (Sequencing Bacth Reactor) la concentración de nitrato en cada ciclo se mantuvo constante y en torno a $50 \mathrm{mg} \mathrm{NO}_{3}-\mathrm{N}^{-1}$. El porcentaje de desnitrificación obtenido fue superior al $60 \%$. El estudio microbiológico realizado, empleando la técnica FISH, mostró en el reactor la presencia de bacterias metanotróficas (tipo I y II), bacterias sulfatoreductoras y bacterias desnitrificantes.
\end{abstract}

Palabras clave: SBR, Desnitrificación, metano disuelto, ácidos grasos, nitratos, SAnMBR.

\begin{abstract}
The treatment or after-treatment of anaerobic effluent suggests study of various processes that allow the elimination or removal of contaminants. The objective of this work consisted in the study of the ability of denitrification under anoxic conditions using different givers of electrons, available in the SAnMBR such as reactor effluent: volatile fatty acids, dissolved methane and sulfur. The importance of this study lies in the possibility of using volatile fatty acids, methane and sulfide dissolved as givers of electrons, for the Elimination of nitrogen. The dissolved methane is a source of very cheap carbon and an effective greenhouse gas, requiring their removal. The SAnMBR reactor effluent contains significant concentrations of ammonium, phosphorus, sulfur, acetic acid and elements dissolved methane and trace. During the operation of the reactor SBR (Sequencing Bacth Reactor) the concentration of nitrate in each cycle remained constant, around $50 \mathrm{mg} \mathrm{NO}_{3}-\mathrm{Nl}^{-1 .}$ The percentage of denitrification retrieved was higher than $60 \%$. The microbiological study, using the FISH technique, showed the presence of bacteria metanotroficas (type I and II), sulfatoreductoras bacteria and denitrifying bacteria in the reactor.
\end{abstract}

Keywords: denitrification, dissolved methane, fatty acids, nitrates, SAnMBR, SBR

1 - MSc. Estudiante doctorado Dpto. Ingeniería Química. U. de Valencia (España).Av de la Universitat,Burjassot. Javier.E.Sanchez@uv.es

2 - PhD profesor Dpto. Ingeniería Quimica. U. de Valencia (España).Av de la Universitat, Burjassot.Alberto.bouzas@uv.es

3 - PhD profesor Dpto. Ingeniería Quimica. U. de Valencia (España), Av de la Universitat, Burjassot. Aurora.seco@uv.es

4- PhD Profesor Dpto. Ing. Hidráulicay Medio Ambiente. U. Politécnica de Valencia (España). IIAMA.Camí de Vera. Magarus@hma.upv.es 


\section{Introducción}

La aplicación de procesos anaerobios para el tratamiento de agua residual urbana se ha convertido últimamente en una tecnología de gran interés debido a sus ventajas en comparación con los procesos aerobios. Sin embargo, el efluente de los procesos anaerobios contiene concentraciones importantes de nitrógeno, fósforo, sulfuro y metano disuelto, siendo necesario su tratamiento. El metano es un gas de efecto invernadero con un potencial de calentamiento global 25 veces mayor que la de dióxido de carbono (Strous, 2010). El sulfuro de hidrógeno es un gas tóxico y corrosivo que causa problemas ambientales y económicos. Los nutrientes como el nitrógeno y el fósforo tienen valores establecidos antes de su vertido.

Uno de los procesos biológicos más utilizados para la eliminación de nitrógeno en las aguas residuales es la desnitrificación. El proceso de desnitrificación transforma el nitrato presente en nitrógeno gas utilizando organismos heterótrofos facultativos 0 autótrofos, en condiciones anóxicas (ausencia de oxígeno y presencia de nitrato). Con este proceso se completa la eliminación biológica de nitrógeno en aguas residuales. La desnitrificación requiere de una fuente de energía, orgánica o inorgánica (dador de electrones), y el nitrato o nitrito como aceptor de electrones. Algunas de las fuentes externas de carbono o dadores de electrones comúnmente encontrados en las aguas residuales son: metanol, etanol, ácido acético y el metano disuelto. Algunos autores mencionan otros compuestos como el sulfuro, que pueden utilizarse en los procesos de desnitrificación (Amaral et al., 1995; Talaso et al., 1997).

La desnitrificación con metano disuelto es una opción interesante en el tratamiento de efluentes provenientes de reactores anaerobios. Algunos autores sugieren que la desnitrificación usando metano disuelto como fuente de carbono se lleva a cabo por una serie de organismos capaces de utilizar el metano como sustrato en condiciones aerobias, anóxicas o anaerobias. En condiciones aerobias, los organismos metanotróficos participan en el ciclo global del carbono, oxidando el metano que ha sido generado en ambientes anaerobios antes de que llegue a la atmósfera. Estos organismos son capaces de utilizar el metano como fuente de carbono y energía. Los organismos metanotróficos más comunes son los de tipo I, II y tipo X. Una característica interesante de estos organismos es la capacidad de utilizar enzimas específicas para catalizar el metano convirtiéndolo en otro compuesto de carbono fácilmente biodegradable como el metanol. Este hecho sugiere que el metano se pueda utilizar como único donante de electrones en la eliminación biológica de nitrógeno específicamente en el proceso de desnitrificación. La literatura sugiere que el metano puede ser transformado por los organismos metanotróficos en condiciones anóxicas y anaerobias en compuestos orgánicos fácilmente biodegradables.

En un trabajo realizado por Beristain-Cardoso et al. (2006) se menciona la posibilidad de realizar el proceso de desnitrificación utilizando sulfuro como dador de electrones y se menciona la posibilidad de realizar la oxidación de los compuestos de azufre hasta sulfato.

El objetivo de este trabajo fue estudiar el proceso de desnitrificación empleando el efluente de un reactor anaerobio de membranas sumergidas (SAnMBR) con el fin de utilizar el metano disuelto, el sulfuro y los ácidos grasos volátiles como dadores de electrones. Este artículo presenta y discute los resultados obtenidos en el estudio a escala laboratorio empleando un reactor SBR operado en condiciones anóxicas. También presenta el estudio microbiológico realizado empleando la técnica FISH.

\section{Materiales y métodos}

\subsection{Reactor SBR}

El estudio a escala laboratorio se realizó en un reactor SBR (Sequencing Batch Reactor), fabricado en metacrilato transparente con forma cilíndrica y con un volumen útil de 7 litros. El reactor fue inoculado con 7 litros de fango biológico procedente de la planta de tratamiento de aguas residuales de la cuenca del Carraixet ubicada en Valencia (España). La parte superior del reactor estaba cerrada con el objetivo de evitar la pérdida de gases y la reaireacción del sistema. Para controlar la temperatura del proceso, el reactor estaba ubicado en una cámara termostática. El reactor disponía de una entrada en la parte inferior para el ingreso del afluente y una salida en la parte lateral intermedia. Para controlar y asegurar los volúmenes máximos y mínimos de agua residual en el reactor el sistema contaba con dos sensores de nivel. El llenado se realizaba por acción de una bomba peristáltica automatizada, y el vaciado por gravedad mediante la apertura de una electroválvula situada en la conducción de salida.

Para dosificar el nitrato $\left(\mathrm{N}-\mathrm{NO}_{3}\right)$ y el ácido acético 
$\left(\mathrm{CH}_{3} \mathrm{COOH}\right)$ al inicio de cada ciclo de operación se utilizaron dos dosificadores de alta precisión modelo Liquino 711 (Metrohm). En la Figura 1 se observa el diagrama general del sistema experimental empleado. La completa homogeneización del fango en el interior del reactor se consigue mediante un agitador mecánico de $50 \mathrm{~Hz}$ y 35 - 2800 rpm (velocidad de giro controlada por un regulador de potencia para minimizar la transferencia de oxígeno desde la atmósfera a la fase líquida del tanque) marca Heidolph. Para monitorizar las variables del proceso se emplearon electrodos de $\mathrm{pH}$, redox, temperatura y oxígeno disuelto (OD). La información de los electrodos era enviada a un software de control y adquisición de datos.
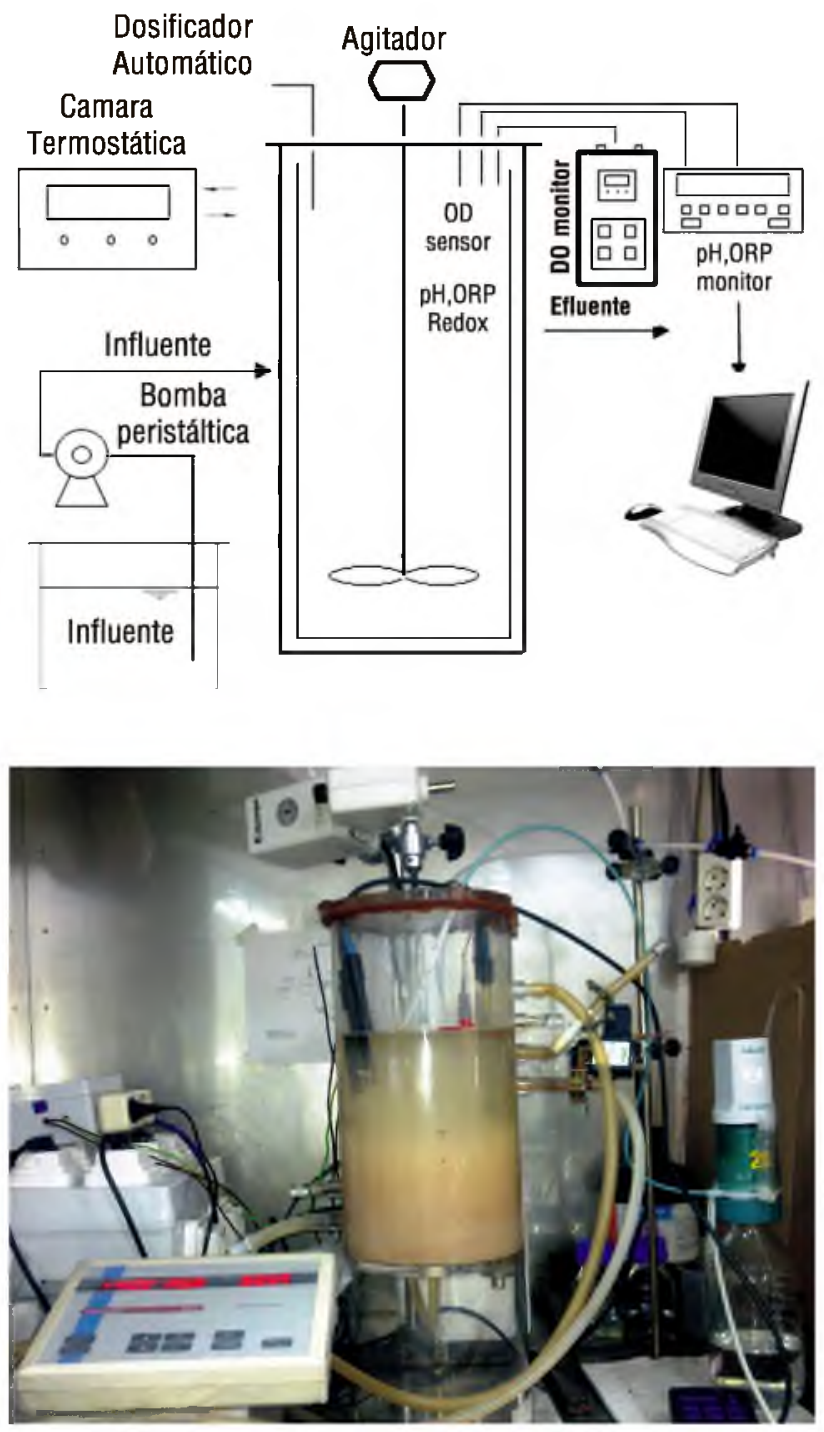

Figura. 1.

Esquema y fotografia del montaje experimental del reactor SBR.

\subsection{Métodos analíticos}

Las técnicas analíticas utilizadas en este trabajo corresponden a las descritas en el Standard Methods (APHA, 2005) para la determinación de sólidos suspendidos totales, sólidos suspendidos volátiles, amonio $\left(\mathrm{N}-\mathrm{NH}_{4}\right)$ y sulfuro $\left(\mathrm{S}-\mathrm{S}^{-2}\right)$. Para la determinación de la alcalinidad y los ácidos grasos volátiles (AGV) se empleó el método de valoración ácido-base propuesto por Moosbrugger et al., 1992. El nitrógeno $\left(\mathrm{N}_{2}\right)$ fue calculado mediante el balance de $\mathrm{N}$ en el sistema. El metano disuelto fue calculado a partir de la medida de metano en el biogás de la planta SAnMBR y el equilibrio entre fases empleando la ley de Henry. El metano en el biogás fue medido empleando un analizador continuo de gases (X-Stream X2, Emerson).

\subsection{Análisis microbiológico}

Las muestras analizadas para la identificación y cuantificación de los microorganismos de interés fueron tomadas del SBR durante la etapa de agitación. Para la observación se utilizó un microscopio de epifluoresencia marca DM2500 con cámara digital DFC420c. En la aplicación de la técnica FISH se emplearon las sondas EUBMIX para la hibridación del dominio eubacteria (Amann et al., 1990; Daims et al., 1999), las sondas Ma464 y Mg84 para la hibridación de los organismos metanotróficos tipo I y II (Eller et al., 2001) y las sondas DEN67 y AT1458 para la identificación de organismos heterótrofos desnitrificantes (Ginige et al., 2005; Rabus et al., 1999).

\subsection{Procedimiento experimental}

Después de inocular el reactor con 7 litros de fango, se fijaron 4 ciclos al día, cada ciclo con una duración total de 6 horas, el tiempo de retención celular fijado (TRC) fue de 20 días y el tiempo de retención hidráulico (TRH) se mantuvo en $19 \mathrm{~h}$.

A continuación se describen cada una de las etapas que componen el ciclo. Etapa de llenado, con una duración total de 6 minutos que equivale al $1.6 \%$ del tiempo total del ciclo, una etapa anóxica con una duración total de 5,2 horas, donde se llevaba a cabo el proceso de desnitrificación y que equivale al $86 \%$ del ciclo total. Una etapa de decantación con una duración total de 33 min equivalente al 9,2\% del tiempo total, en esta etapa la agitación cesaba y se dejaba decantar el fango. Finalmente, se procedía a la etapa de vaciado del sobrenadante con una duración total de $6 \mathrm{~min}$, 
equivalente a un $1,6 \%$ del tiempo total del ciclo quedando el reactor listo para un nuevo ciclo Figura 2. La distribución de tiempos se realizó teniendo en cuenta las experiencias obtenidas en otros estudios, desarrollados en el grupo de investigación (Acevedo B etal., 2012; Aguado Detal., 2009). A
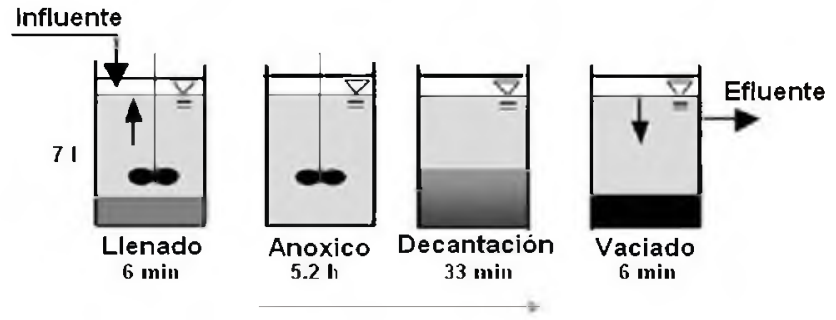

Figura. 2. Etapas en cada ciclo de operación.

\section{Resultados}

El reactor SBR se operó durante 6 meses en condiciones anóxicas. El seguimiento de los compuestos de nitrógeno presentes en el sistema permitió observar eficiencias en la remoción de nitrógeno superiores al $\mathbf{8 0 \%}$. El efluente del reactor SAnMBR alimentado al SBR, presentaba una baja DQO biodegradable (ca. $30 \mathrm{mg}$ DQO $\cdot \mathrm{L}^{-1}$ ), elevadas concentraciones de nutrientes $\left(55 \mathrm{mg} \mathrm{N}-\mathrm{NH} 4 \cdot \mathrm{L}^{-1}, 7\right.$ $\mathrm{mg} \mathrm{P}-\mathrm{PO}_{4} \cdot \mathrm{L}^{-1}, 105 \mathrm{mg} \mathrm{S} \cdot \mathrm{L}^{-1}$ ) y una concentración de metano disuelto en torno a $43 \mathrm{mg} \mathrm{DQO} \cdot \mathrm{L}^{-1}$. Para este estudio teniendo en cuenta que el nitrógeno presente en el efluente se encontraba en forma de amonio $\left(\mathrm{N}-\mathrm{NH}_{4}\right)$, se adicionó al efluente un patrón de nitrato para una concentración en el alimento de $50 \mathrm{mg}$ $\mathrm{N}-\mathrm{NO}_{3} \cdot \mathrm{L}^{-1}$. Esta concentración al inicio de cada ciclo corresponde a $15 \mathrm{mg} \mathrm{N}-\mathrm{NO}_{3} \cdot \mathrm{L}^{-1}$ en el reactor. Las especies desnitrificantes presentes en el inóculo fueron identificadas con las sondas DEN67, PAR651 y AT1458. La abundancia de estos organismos fue de un $4 \pm 2 \%$ (DEN67), $1 \pm 1 \%$ (PAR651) y $8 \pm 1 \%$ (AT 1458) respectivamente. Los organismos metanotróficos tipo I y II presentaron una abundancia del $1 \%$. Este estudio fue dividido en cinco periodos, a lo largo de la cuales se fue modificando el TRC. La Tabla 1 resume los cambios realizados y el porcentaje de desnitrificación obtenido.
Tabla 1. Condiciones de operación del reactor SBR.

\begin{tabular}{lclll}
\hline Periodo & \% Desnitrificación & TRH (h) & TRC(d) & T $\left({ }^{\circ} \mathbf{C}\right)$ \\
\hline I & 24,6 & 19,0 & - & 20,0 \\
II & 69,0 & 19,0 & 35 & 20,0 \\
III & 86,0 & 19,0 & 25 & 20,0 \\
IV & 87,8 & 19,0 & 30 & 20,0 \\
V & 93,6 & 19,0 & 20 & 20,0 \\
\hline
\end{tabular}

La Figura 3 muestra la evolución de la concentración de nitrato en cada periodo. Tal y como se observa en la figura el nitrato presente al inicio de cada ciclo se reduce a nitrógeno gas es cual es calculado a partir del balance de nitrógeno en el sistema. Este hecho confirma que existe una población de organismos desnitrificantes capaces de desnitrificar los compuestos de nitrógeno presentes en forma de nitrato o nitrito. Al final del periodo I se observa una etapa de transición en el que la concentración de nitrato comenzó a descender. Durante esta etapa de transición se ajustó de forma escalonada el TRC. Tras el periodo de transición se observan diversos periodos de estabilidad en función del TRC estudiado. En la Figura 3 también se observa que inicialmente el sistema se dejó evolucionar con el objetivo de adaptar los organismos presentes a las características del afluente y favorecer el crecimiento de los organismos desnitrificantes. Durante el periodo I el porcentaje de desnitrificación se situó en torno al $24 \%$.

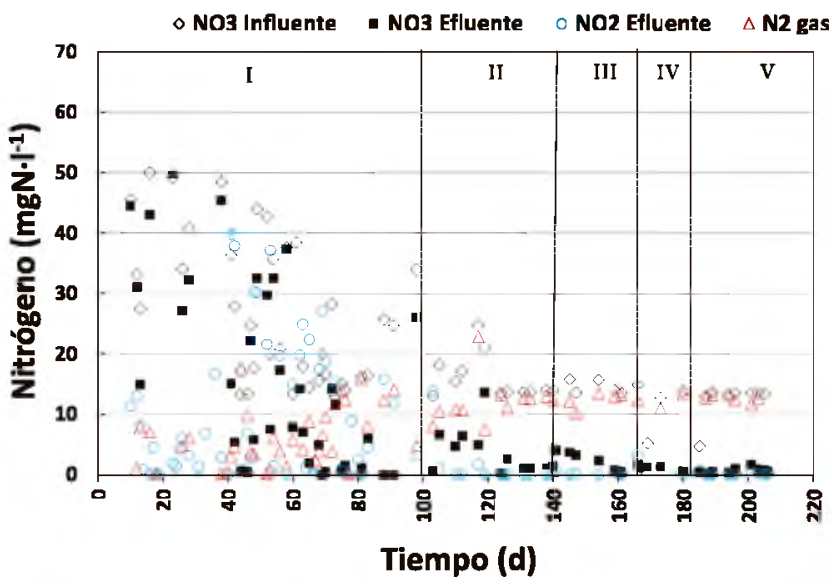

Figura. 3. Evolución del nitrato y nitrógeno gas durante la operación del reactor SBR.

La abundancia de organismos desnitrificantes se situó en un $24 \%$ indicando un aumento importante de estos microorganismos. Otro aspecto importante observado en el estudio microbiológico realizado en este periodo fue el aumento de la abundancia de organismos metanotróficos tipo I (4\%), este crecimiento está aso- 
ciado al consumo de metano disuelto. En los periodos II, III, IV y V el porcentaje de desnitrificación alcanzado fue del $69,86,87$ y $93 \%$ respectivamente.

Durante los periodos III, IV y V se observó un aumento considerable de los AGV (100 mg DQO $\cdot \mathrm{L}-$ 1) que podría haber favorecido el proceso de desnitrificación. Sin embargo en los periodos I y II la DQO en forma de AGV fue baja y los porcentajes de desnitrificación alcanzados fueron del 24 y $69 \%$ respectivamente. Este hecho sugiere que podrían existir otros compuestos presentes en el efluente que participan en la desnitrificación actuando como dadores de electrones $\left(\mathrm{S}^{2-} \mathrm{y} \mathrm{CH}_{4}\right.$ disuelto).

Con el fin de comprobar la participación de estos compuestos se realizó un balance de DQO en el sistema teniendo en cuenta la concentración de sulfuro y metano disuelto. Los resultados obtenidos ponen de manifiesto que en los periodos I y II la materia orgánica fácilmente biodegradable (AGV) no era suficiente para alcanzar los porcentajes de desnitrificación obtenidos, por tanto existen en el afluente otros dadores de electrones que pueden contribuir en el proceso de desnitrificación, tales como el metano disuelto y sulfuro (Tabla 2).

Tabla 2. Concentración de AGV, $\mathrm{CH}_{4}, \mathrm{~S}^{2}$ y nitrato en cada periodo.

\begin{tabular}{|c|c|c|c|c|c|c|}
\hline Periodo & $\begin{array}{c}\text { AGV } \\
\left.(\mathrm{mg} \mathrm{DQO.1})^{-1}\right)\end{array}$ & $\begin{array}{c}\text { CH4 } \\
\left.(\mathrm{mg} \mathrm{DQO.1})^{-1}\right)\end{array}$ & $\begin{array}{c}\mathrm{C}^{2-} \\
\text { (mg DQO.1 }\end{array}$ & $\begin{array}{c}\mathrm{N}-\mathrm{NO}_{3} \text { teorico } \\
\left(\mathrm{mgN} \cdot \mathbf{1}^{-1}\right)\end{array}$ & $\begin{array}{c}\mathrm{N}-\mathrm{NO}_{3} \text { eliminado } \\
\left(\mathrm{mgN} . \mathbf{1}^{-1}\right)\end{array}$ & \% Desnitrificación \\
\hline I & 14,0 & 6,9 & 14,2 & 4,86 & 6,5 & 24,0 \\
\hline II & 5,0 & 4,5 & 13,1 & 1,74 & 11,6 & 69,0 \\
\hline III & 61,0 & 12,8 & 16,7 & 21,3 & 12,3 & 86,0 \\
\hline IV & 108,0 & 1,5 & 20,4 & 37,7 & 12,7 & 87,0 \\
\hline V & 40.0 & 6,4 & 8,2 & 13.9 & 12.6 & 93.0 \\
\hline
\end{tabular}

El estudio microbiológico realizado empleando la técnica FISH permitió observar la presencia de organismos heterótrofos desnitrificantes, metanotróficos y sulfatoreductores durante cada periodo de operación. La Tabla 3 muestra la abundancia de cada organismo durante cada periodo. Se puede observar que la especie más abundante corresponde con los organismos heterótrofos desnitrificantes, hibridados con la sonda AT1458 capaces de usar los ácidos grasos volátiles como fuente de carbono.

Durante el seguimiento microbiológico también se realizó el seguimiento de las especies sulfatorreductoras y organismos del tipo thiobacilus desnitrificantes debido a la presencia de sulfuro y sulfato en el sistema. En el caso de los organismos thiobacilus desnitrificantes no fueron detectados. También se realizó la identificación y cuantificación de las bacterias metanotróficas capaces de utilizar metano disuelto para su crecimiento (Figura 4).

Tabla 3. Abundancia de organismos desnitrificantes, metanotróficos y sulfatoreductores.

\begin{tabular}{ccccc}
\hline ABUNDANCIA \% \\
\hline Periodo & Desnitrificantes & $\begin{array}{c}\text { Metanotróficas } \\
\text { tipo I }\end{array}$ & $\begin{array}{c}\text { Metanotróficas } \\
\text { tipo I }\end{array}$ & $\begin{array}{c}\text { Sulfato } \\
\text { reductoras }\end{array}$ \\
\hline I & $11 \pm 1,0$ & $1 \pm 1,0$ & ND & $5 \pm 1,0$ \\
II & $26 \pm 3,0$ & $5 \pm 1,0$ & ND & $2 \pm 1,0$ \\
III & $30 \pm 7,0$ & $8 \pm 2,0$ & $1 \pm 1,0$ & $6 \pm 1,0$ \\
IV & $24 \pm 5,0$ & $3 \pm 1,0$ & $2 \pm 1,0$ & $7 \pm 1,0$ \\
V & $26 \pm 4,0$ & $1 \pm 1,0$ & $1 \pm 1,0$ & $1 \pm 1,0$ \\
\hline
\end{tabular}



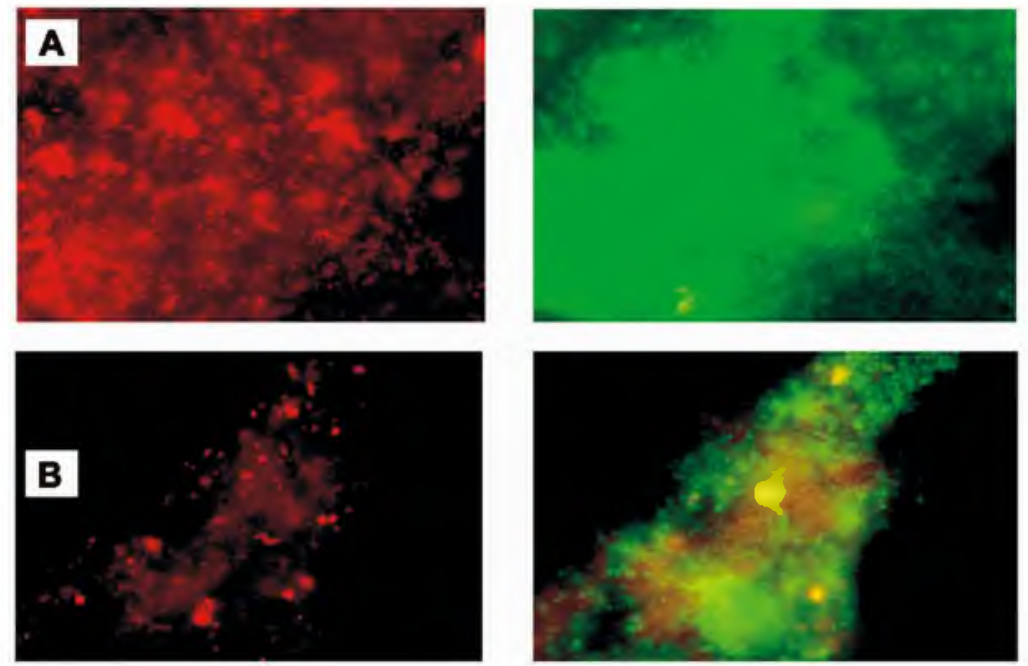

Figura. 4. FISH, Fotos capturadas con el microscopio de epifluorecencia, (A) bacteria desnitrificantes (rojo), (B) Bacterias metanotróficas (rojo), Total de bacterias hibridadas con la sonda EUBmix (verde).

La desnitrificación con sulfuro se corroboró mediante ensayos off-line realizados en laboratorio. La Figura 5 muestra la evolución de la concentración de nitrato en presencia de sulfuro en uno de los ensayos realizados. Se observa que el sulfuro es oxidado rápidamente a compuestos como el tiosulfato $\left(\mathrm{S}_{2} \mathrm{O}_{3}\right)$ y el sulfato $\left(\mathrm{SO}_{4}\right)$. También se observó que mientras la concentración de nitrato decrece, la concentración de nitrito aumenta y pasa a nitrógeno gas, debido al proceso de desnitrificación. En otros ensayos se pudo corroborar que si existe sulfuro en el sistema, los nitritos son reducidos en su totalidad a nitrógeno gas $\mathrm{N}_{2}$. Por lo que respecta al metano disuelto, el aumento en la abundancia de los organismos metanotróficos (tipo I y Tipo II) en el sistema permite concluir que el metano disuelto es utilizado como sustrato por estos organismos.

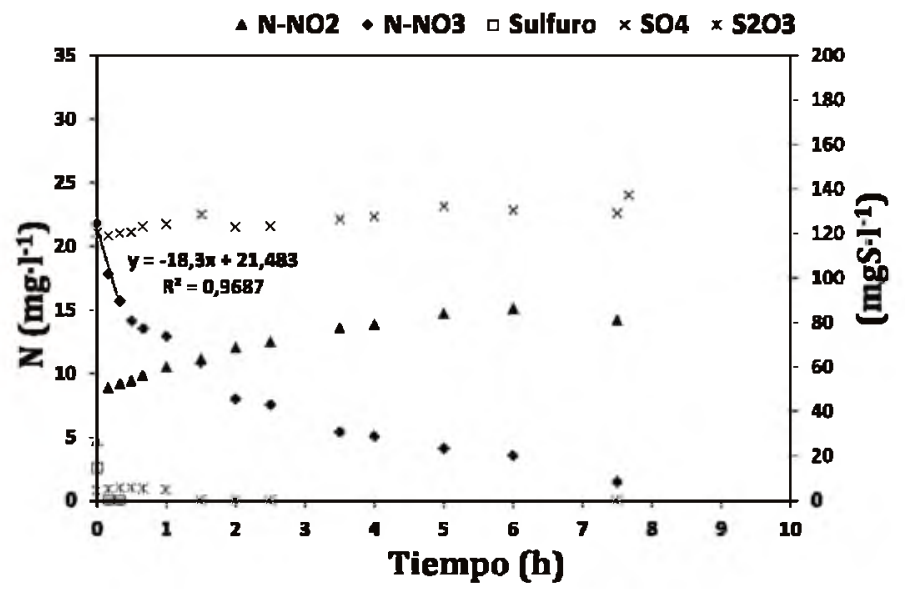

Figura. 5. Evolución del nitrato y nitrógeno gas durante la operación del reactor SBR.

\section{Conclusiones}

En general, el estudio a escala laboratorio permitió determinar que es posible obtener rendimientos en la desnitrificación mayores al $90 \%$ utilizando los dadores de electrones presentes en el afluente. También fue posible observar que existen diversos dadores de electrones como el sulfuro o el metano disuelto, que pueden aportar DQO al proceso $y$ contribuir en la desnitrificación. El crecimiento observado mediante la técnica FISH de los organismos metanotróficos tipo I y tipo II pone de manifiesto el uso de metano disuelto como sustrato por parte de estos organismos.

Los resultados obtenidos en el seguimiento del sistema en cada periodo mostraron que utilizando solamente los AGV no sería posible alcanzar el grado de desnitrificación obtenido en cada periodo. Tal y como se ha expuesto anteriormente, el sulfuro y el metano disuelto podrían estar participando como dadores de electrones en la desnitrificación, con lo cual en este estudio estos compuestos representan un aporte importante de DQO.

\section{Agradecimientos}

Se agradece la financiación al ministerio de ciencia e innovación (proyecto CTM2011-28595-C02 01/02) y a la Universidad de Valencia (proyecto precompetitivo UV-INV-AE11-40539). 


\section{Referencias Bibliográficas}

1. Acevedo, B., Oehmen, A., Carvalho, G., Seco, A., Borrás, L., and Barat, R. (2012). Metabolic shift of polyphosphate-accumulating organisms with different levels of polyphosphate storage. Water research, 46(6), 1889-1900.

2. Aguado, D., Ribes, J., Montoya, T., Ferrer, J., and Seco, A. (2009). A methodology for sequencing batch reactor identification with artificial neural networks: a case study. Computers \& Chemical Engineering, 33(2), 465-472.

3. Amann, R.I.,(1995). Fluorescently labelled, rRNAtargeted oligonucleotide probes in the study of microbial ecology. Molecular Ecology. 4 (5), 543 554.

4. Amaral, J.A., Archambault C., Richards, S.R., Knowles, R., (1995). Denitrification associated with groups I and II methanotrophs in a gradient enrichment system. FEMS Microbiol. Ecol. 18, 289298

5. APHA/AWWA, (2005). Standard Methods for the Examination of Water and Wastewater, 21 th ed. American Public Health Association, American Water Works Association and Water Environment Federation, Washington DC, USA.

6. Costa, C., Dijkema, C., Friedrich, M., García-Encina, P., Fernandez - Polanco, F., Stams, A.J.M., (2000). Denitrification with methane as electron donor in oxygen limited bioreactors. Appl. Microbiol. Biotechnol. 53, 754-762.

7. Daims, H., Bruhl, A., Amman,R., Schleifer,K.H., Wagner, M.,(1999). The domain specific probe EUB 338 is insufficient for the detection of all bacteria: development and evaluation of a more comprehensive probe set. Syst. Appl. Microbiol. 22, 434-444.

8. Eller, G., Stubner, S., Frenzel, P., (2001). Groupspecific 16S rRNA targeted probes for the detection of type I and type II methanotrophs by fluorescence in situ hybridization. FEMS Microbiol. Lett. 198, 91-97.
9. Hatamoto, M., Miyauchi, T., Kindaichi, T., Ozaki, N., Ohashi, A., (2011).Dissolved methane oxidation and competition for oxygen in down-flow hanging sponge reactor for post-treatment of anaerobic wastewater treatment, Bioresource Technology. 08, 99.

10. Moosbrugger, R. E., Wentzel, M. C., Ekama, G. A., Marais, G. v. R., (1992). Simple titration procedures to determine $\mathrm{H} 2 \mathrm{CO}^{*}$ alkalinity and short-chain fatty acids in aqueous solutions containing known concentrations of ammonium, phosphate and sulphide weak acid/bases. Water Research Commission, Report No. TT 57/92. University of Cape Town, Research Report W 74, Pretoria, Republic of South Africa.

11. Modin, O., Kensuke, F., Kazuo, Y., (2007). Denitrification whit methane as external carbon source. Water research 41, 2726 - 2738.

12. Islas-lima, S., Thalasso, F., Gómez-Hernandez, J., (2004). Evidence of anoxic methane oxidation coupled to denitrification. Water Research. 38, 13-16.

13. Rabus, R., Wilkes, H., Schramm, A., Harms, G., Behrends, A., Amann, R., Widdel, F.,(1999). Anaerobic degradation of alkylbenzenes and $n-$ alkanes from crude oil in an enrichment culture of denitrifying bacteria affiliating with the ?-subclass of Proteobacteria. Environmental Microbiology (2),145157.

14. Souza, C.L., Chernicharo, C.A.L., Aquino, S.F., (2010). Quantification of dissolved methane in UASB reactor treating domestic wastewater under different operating conditions. IWA world congress on anaerobic digestion. Guadalajara.

15. Strous, M., (2010). Global consequences of anaerobic methane oxidation. Handbook of Hydrocarbon an lipid Microbiology. 3078 - 3083.

16. Thalasso, F., Vallecillo, A., García - Encina, P., Fernandez- Polanco, F.,(1997). The use of methane as a sole carbon source for wastewater denitrification. Water Res. 31(1), 55 